\title{
Strategies for mature T cell cancers
}

Cancers of mature T cells, such as T cell non-Hodgkin lymphoma (NHL), are aggressive and resistant to treatment. Two new papers provide insight into the future treatment of mature $\mathrm{T}$ cell cancers. Wartewig and colleagues demonstrate that PDCD1, which encodes programmed cell death protein 1 (PD1), is a haploinsufficent tumour suppressor in this cancer, suggesting that immunooncology strategies inhibiting PD1 could, unlike in other tumours, cause T cell NHLs to expand. In a second paper, Maciocia and colleagues exploit the clonal nature of tumours to specifically target one of the T cell receptor $\beta$-chain constant domains, TRBC1, using a chimeric antigen receptor (CAR) T cell approach.

Wartewig and colleagues began by generating mice that express a transgene that encodes a fusion protein, ITK-SYK, which is produced by a translocation found in deletion of one

or both copies

of PDCD 1

reduced the

time to the

formation

of lethal

infiltrative

lymphomas

5

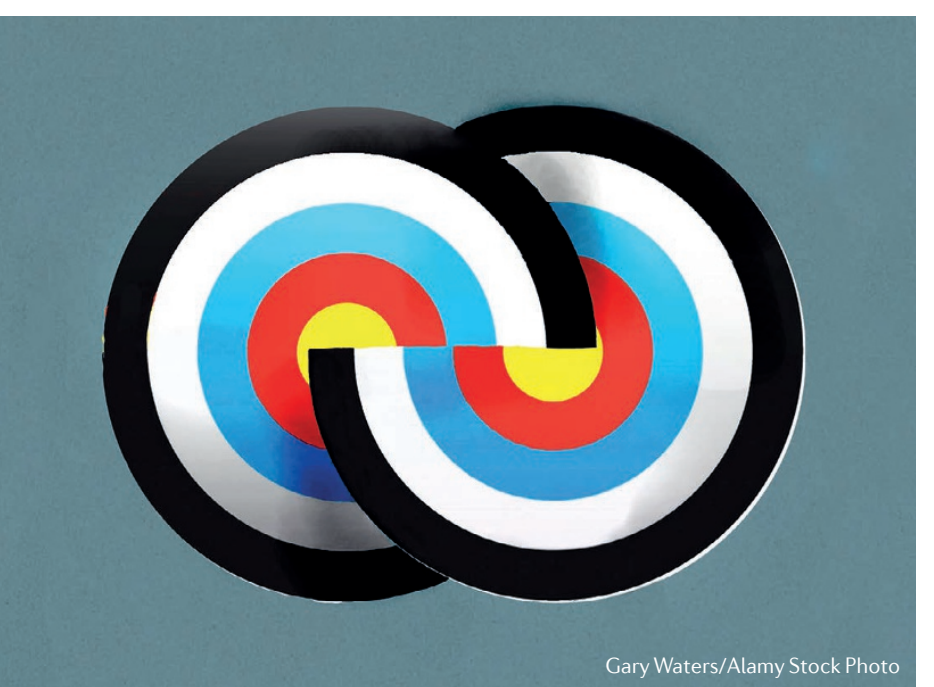

human T cell NHL. ITK-SYK has constitutive tyrosine kinase activity and promotes oncogenic signalling from T cell receptor (TCR) pathways. Expression of the ITK-SYK transgene in $\mathrm{CD}^{+}$cells resulted in fully penetrant, aggressive, clonal $\mathrm{T}$ cell lymphomas after 200-250 days, following an initial cell expansion and contraction phase.

This delay in tumorigenesis suggested that additional alterations are required. Using a transposon mutational screen, the authors identified PDCD1 as the most common gene that, if disrupted, leads to rapid transformation. Indeed, in a meta-analysis of five published studies of human $\mathrm{T}$ cell lymphomas, $P D C D 1$ was altered in 36 of 158 cases.

In mice that express ITK-SYK in $\mathrm{T}$ cells, deletion of one or both copies of $P D C D 1$ reduced the time to formation of lethal infiltrative lymphomas to about 1 week $\left(P d c d 1^{-/}\right)$or 1 month $\left(P d c d^{+/}\right)$. Similarly, treating mice expressing ITK-SYK with inhibitors of PD1 or programmed death 1 ligand 1 (PDL1), a recently established class of immuno-oncology agent, led to an immediate and lethal expansion of ITK-SYK ${ }^{+}$cells.

In $\mathrm{T}$ cell lines, ITK-SYK drives PD1 expression. Normally, PD1 ligation dampens oncogenic signalling by increasing levels of phosphatase and tensin homologue (PTEN), thus providing a negative feedback loop: PTEN counteracts the effects of phosphoinositide 3-kinase (PI3K), a key downstream component of TCR signalling that is engaged by ITK-SYK. Treatment of ITK-SYK-expressing mice with the PI3K inhibitor idelalisib significantly extended their lifespan, underscoring the importance of this signalling pathway and also providing a potential therapeutic avenue.

In another paper, Maciocia and colleagues generated CAR T cells that target TRBC1, but not TRBC2, to treat mature $\mathrm{T}$ cell cancers. Each $\mathrm{T}$ cell (and therefore each T cell cancer) irreversibly selects either TRBC1 or TRBC2 to incorporate into TCRs. Approximately 35\% of normal and virus-specific $\mathrm{T}$ cells express TRBC1, and 65\% express TRBC2. Thus, targeting tumours that express TRBC1 should deplete the tumour cells but leave enough $\mathrm{T}$ cells to fight infections.

T cells transduced with TRBC1specific CAR constructs were able to kill TRBC1 ${ }^{+}$cell lines, and allogenic or autologous CAR T cells were able to kill $\mathrm{TRBC}^{+}$primary human malignant cells but not normal TRBC2 ${ }^{+}$ T cells. These CAR T cells were also effective in immunodeficient mice engrafted with $\mathrm{TRBC}^{+} \mathrm{T}$ cells. A clinical trial of anti-TRBC1 CAR T cells for $\mathrm{TRBC}^{+}$mature $\mathrm{T}$ cell malignancies is due to commence in 2018.

These findings suggest new therapeutic avenues for the treatment of mature $\mathrm{T}$ cell malignancies, and also caution against the use of PD1 or PDL1 inhibitors in these cancers.

Megan Cully

ORIGINAL ARTICLES Wartewig, T. et al. PD-1 is a haploinsufficient suppressor of T cell lymphomagenesis. Nature http://dx.doi. org/10.1038/nature24649 (2017) | Maciocia, P. M. et al. Targeting the T cell receptor $\beta$-chain constant region for immunotherapy of T cell malignancies. Nat.Med. http://dx.doi.org/10.1038/nm.4444 (2017) 